\title{
Genetic diversity of Asian water buffalo (Bubalus bubalis): microsatellite variation and a comparison with protein-coding loci
}

\begin{abstract}
Twenty-one microsatellite loci in 11 populations of Asian water buffalo (eight swamp, three river type) were analysed and, within and among populations, genetic variability was compared with results from 25 polymorphic protein-coding loci. Within-population mean heterozygosity ranged from $0 \cdot 380 \mathrm{i} 0.615$, approximately twice that estimated from the protein-coding loci $(0 \cdot 184 i ̈$ 0 346). Only eight significant departures from Hardyï Weinberg equilibrium (involving four loci) were detected; global tests showed significant heterozygote deficiencies for these four loci. Non-amplifying alleles are likely to be segregating in some or all populations for one of these loci, and probably for the other three. There was significant differentiation between the swamp and river types of water buffalo, and among populations within each buffalo type. Estimates of $d$ (measure of population differentiation) for each locus for the eight swamp populations were all highly significant (mean $\delta=0 \cdot 168 \pm 0 \cdot 018$ ). Mean $\mathfrak{d}$ for protein-coding loci was not significantly different $(0 \cdot 182 \pm 0 \cdot 041)$. The variance among protein-coding loci was significantly higher than among microsatellite loci, suggesting balancing selection affecting allele frequencies at some protein-coding loci. Genetic distances show clear separation of the swamp and river types, which were estimated to have diverged at least 100001015000 years ago. The topology of the swamp populationsô microsatellite tree is consistent with their geographical distribution and their presumed spread through south-east Asia. By contrast, the tree based on the protein-coding loci distances is quite different, being clearly distorted by a bottleneck effect in one population, and possibly in at least two others. As many domestic livestock breeds are possibly descended from small numbers of founders, microsatellite-based trees are to be preferred in assessing breed genetic relationships.
\end{abstract}

Keyword: Allozymes; Asian water buffalo; Bubalus bubalis; Genetic diversity; Microsatellites; Molecular phylogenetics 